\title{
Characterising Distributed Haptic Virtual Environment Network Traffic Flows
}

\author{
K.M. Yap ${ }^{1}$, A Marshall $^{1}, \mathrm{~W} \mathrm{Yu}^{1}$, G Dodds ${ }^{1}, \mathrm{Q} \mathrm{Gu}^{1}$, Rima T'faily \\ Souayed $^{2}$ \\ ${ }^{1}$ Virtual Engineering Centre \\ Northern Ireland Technology Centre \\ School of Electrical \& Electronic Engineering \\ Queen's University Belfast \\ Phone: - +442890975588 Fax:- +442890974332 \\ Email:-m.yap@qub.ac.uk,A.Marshall@ee.qub.ac.uk,w.yu@qub.ac.uk, \\ \{G.Dodds, Qiang.gu\}@ee.qub.ac.uk \\ ${ }^{2}$ Université de Technologies de Troyes, \\ \{Rima.Tfaily,dominique.gaiti\}@utt.fr
}

\begin{abstract}
The effective transmission of haptic data in Distributed Haptic Virtual Environment (DHVEs) is a new research area which presents a number of challenges to the underlying network. The transmission of reflected force in these applications has the potential to change the way humans interact with machines and communicate with each other. The future Internet will have to carry multiple DHVE type traffic and it is now established that the best effort service offered by current IP networks is insufficient to meet the needs of these type of applications, which require specific Quality of Service (QoS) from network. The aim of the work presented here is to characterise the traffic generated by multiple DHVE network connections. The approach taken is to develop a simulation model of DHVE traffic based on empirical measurements. Both synchronous and asynchronous real world DHVE traffic is analyzed, quantified and imported into a network simulation. Probability Density Function (PDF) models are subsequently derived for each type of traffic in the DHVE system. The results show the network simulation model compares favourably with the physical network, and can be used to generate a scalable haptic network model where multiple DHVE connections may be examined.
\end{abstract}

Please use the following format when ciling this chapter:

Yap, K.M., Marshall, A., Yu, W., Dodds, G., Gu, Q. and Souayed, R.T., 2007, in IlIP International Federation for Information Processing, Volume 229, Network Control and Engineering for QoS, Security, and Mobility, IV, ed. Gaitti, D., (Boston: Springer), pp. 297-310. 
Keywords: haptic, distribution virtual environment, traffic characteristics, tele-operation, network simulation.

\section{Introduction}

3D virtual environment technologies have been used in numerous research areas. The computer graphics, industry, hazardous industries as well as tele-robotic, education training and interactive advertisement are some areas of application. By definition, a virtual environment (VE) is a space that provides users with the illusion of acting in a real world [12]. However in addition to audio and visual information, the provision of haptic feedback (the sense of touch) can profoundly improve the way we interact with virtual environments. Systems that support interfaces between a haptic device and a virtual environment are called Haptic Virtual Environments (HVEs). HVE uses include military and space exploration; the sense of touch will also enable blind people to interact with each other within a virtual environment. The HVE modalities include graphics (and possibly video), sound and force. Recent research [2] has shown that to have a satisfying experience in interacting with a HVE, the graphics and haptic update rates need to be maintained at around $30 \mathrm{~Hz}$ and $1 \mathrm{KHz}$ respectively. HVEs can be standalone or distributed. In a standalone HVE, both the haptic virtual environment and the haptic device reside on, or are connected to the same machine. In distributed HVEs (DHVE) otherwise known as tele-haptic systems, the haptic device is separate from the virtual environment and remotely affects and manipulates it. In DHVEs, one or multiple users may interact with the virtual environment, and possibly with other users with haptic devices. Users may take turns in manipulating a virtual object as in Collaborative Environments or may simultaneously modify the same object as in, for example, Cooperative Environments [13]. The DHVE provides the feeling of tele-presence for a single user and the feeling of both tele-presence and co-presence for multiple users.

The effect of network impairments has a direct impact on the sense of human perception during DHVE interactions [1]. Each network impairment affects the sense of force feedback in a particular way. For example, considerable network delay may make the user feels a virtual object either before or after they interact with it on the virtual environment (i.e. it is possible to go through a virtual wall before feeling it). Delay also desynchronizes the different copies of the virtual environment. Packet delay variation (jitter) makes the user feel that the object's mass is variable, and can make the system unstable (e.g. it can produce oscillations on the sur- 
faces of objects). Network QoS performance is generally described using four basic parameters. These are: (i) Throughput: the number of packets that can be transmitted in a fixed amount of time. (ii) Delay: the difference between the time when the packet has been sent and the time when it is received. (iii) Jitter: the statistical variance of delay measured as the average time between two successively received IP packets. (iv) Packet Loss: expressed as a percentage of the number of packets not received, to the number of packets sent. Packet loss can reduce the amount of force felt by the user. A major challenge is therefore to clarify the QoS requirements of collaborative haptic systems such as DHVEs when they are considered for use over the Internet. In particular, the effects of network impairments upon virtual environments need to be quantified. Additionally, an investigation into the suitability of aggregated QoS architectures such as DiffServ [14], in supporting multiple DHVE-type collaborations is required. The work presents in this paper empirically models and characterises DHVE traffic so that scalable haptic QoS architectures may be developed.

\section{An Experimental Testbed for Characterising DHVE traffic}

In order to examine what multiple DHVE traffic flows look like, it is first necessary to characterise individual DHVE connections. The approach taken here is to set up a test network and run various DHVE applications over it. The collected network traces are then used to generate statistical models of each type of DVHE traffic that can be used in standard network modelling packages such as OPNET [11].

\subsection{Haptic Device}

The force-feedback haptic device used in the experimental testbed is a PHANToM (Fig.1) which is single point contact haptic device by SenSAble Technologies [4]. The PHANToM's interface main component is a serial feedback arm that ends with a stylus, allowing users to feel interaction forces they would encounter while touching objects. It is capable of 6 degrees of freedom input (measurement) and 3 degrees of freedom output (force feedback). It has three motors, allowing translational force feedback. The force feedback is calculated in proportion to the depth of penetration of the PHANToM cursor into a virtual object. The stylus orientation is passive, so no torque can be applied to the user's hand. 


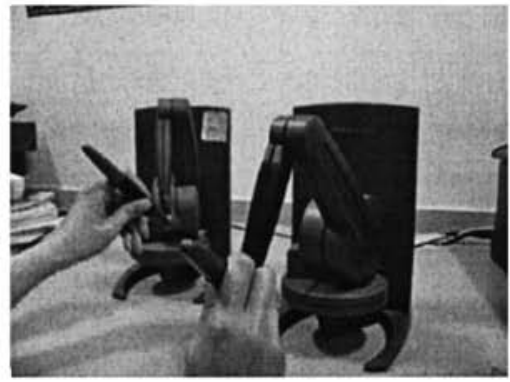

Fig. 1 PHANToM Desktop Devices, Haptic Traffic Generation Devices

The PHANToM can track the position and the orientation of its stylus in an arm workspace of $16 \mathrm{~cm}$ wide, $13 \mathrm{~cm}$ high and $13 \mathrm{~cm}$ deep. The maximum exerted force is $6.4 \mathrm{~N}$, while continuous force is $1.7 \mathrm{~N}$. Its maximum stiffness is high $\left(3^{*} 103 \mathrm{~N} / \mathrm{m}\right)$ to allow realistic simulation of contact with walls and hard objects. The 1000 packets per second sending rate from the PHANToM is greater than that for conventional multimedia applications such as Video, PCM audio or IP telephony, which can range from 30 up to 250 packets per second (depending on the packet frame size used).

\subsection{Synchronous and Asynchronous DHVE Test Applications}

In order to collect DHVE traffic it is first necessary to run DHVE applications over the test network. To this end, two different DHVE test applications were developed that consider synchronous and asynchronous operation respectively. Both systems work in a client-server arhcitecture. In synchronous operation the local position at the client is only updated upon feedback from the server, while with asynchronous operation the local client updates occur regardless of the feedback data. In synchronous operation, the local client sends local PHANToM position to server and waits to receive the feedback position. In the asynchronous operation, the local client will keep sending new PHANToM position data regardless of the previous position.

\subsection{Experiment Configuration}

The experimental set-up involves 2 computers that are connected through a gigabit Ethernet fibre optic link running on best effort IP service. Fig.2 shows the configuration of the experimental network. 


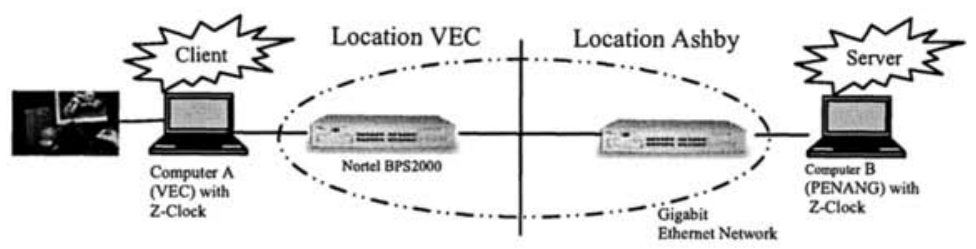

Fig. 2. Experiment Test Bed Setup

In operation, computer A running the client program connects to the PHANToM and generates haptic traffic for computer B which is running the server program. When running, the user holding the PHANToM will experience that he or she is rendering inside a virtual box. The stylus movement is limited by the box's six virtual walls and is unable to go beyond the virtual walls.

\subsection{Traffic Collection and Analysis}

The network traffic is captured by using a software capturing tool called IP Traffic [3]. The measured network parameters are throughput, packet lost, delay and jitter. The captured traffic is analyzed, imported and customized in simulation software packages called OPNET ACE and Modeler [11]. The captured haptic traffic is converted into tcpdump format before imported into OPNET ACE. Subsequently, OPNET ACE shows the haptic packet format and haptic traffic pattern. OPNET PDF models are customized from captured haptic traffic and implemented in OPNET Modeler simulation network model. The reason for using real-world traffic in the simulator is to gain an accurate baseline haptic model that can be used to generate a better representation of a network's performance when supporting this type of traffic.

\section{Experimental Results}

\subsection{Asynchronous Mode Haptic Traffic Packet and Pattern Distribution}

It is important to study what the haptic traffic distribution looks like in order to customize the haptic traffic in the OPNET model. The haptic systems generate 1000 packets/sec and 980 packets/sec in asynchronous and synchronous mode respectively. In asynchronous mode, the data field of a 
haptic packet is 64 bytes, adding IP, UDP and Ethernet header give a total length of 106 bytes without preamble and CRC header. The length of the data field of a synchronous mode packet haptic packet is 40 because less information is transmitted in this mode (i.e. only the PHANToM's positional data is sent); the total network packet size is 82 bytes without preamble and CRC header. The packet size of the haptic traffic is constant but the inter-packet delay varies. Fig. 3 shows the asynchronous mode interpacket delay from client to server. The synchronous mode results are almost identical to the asynchronous mode and are not shown.

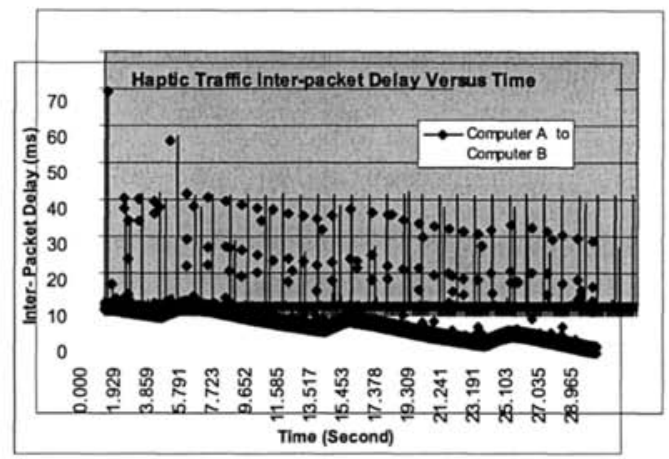

Fig. 3. Asynchronous Mode Haptic Traffic Inter-packet Delay versus time, Client to Server

In Fig.3, it may be observed that most of the inter-packet delays are below $10 \mathrm{~ms}$ which occur at $1.9 \mathrm{~ms}$ and $0.1 \mathrm{~ms}$ with some inter-packet delays experienced at $20 \mathrm{~ms}$ and $30 \mathrm{~ms}$. The maximum inter-packet delay occurs at $59.2 \mathrm{~ms}$. There are also a significant number inter-packet delays near zero. These are due to the nature of the steady $1 \mathrm{~ms}$ time interval of the haptic source application, and after a long inter-packet delay (e.g. $20 \mathrm{~ms})$ there are many packets that have been queued waiting to be sent and are then be burst onto the network at rates faster than $1 \mathrm{~ms}$. This gives an abrupt movement effect to the user; strategies to optimise the performance of haptic applications over bursty traffic flows are the subject of [1]. The interpacket delay from Server to Client shows a larger variation than from Client to Server. This is due to the response time of the server computer. The experiment results showed that the server to client inter-packet delay distribution is similar to the client to server packet distribution. The first packet from the server to client occurs $0.7 \mathrm{~s}$ after the first packet from the client because the server needs time to respond to the haptic traffic from client. The inter-packet delay statistics for both directions are summarised below. 
Table 1. Haptic Traffic Inter-packet Delay Summary

\begin{tabular}{lll}
\hline & Client to Server (ms) & Server to Client (ms) \\
\hline Average $=$ & 0.999996709 & 0.998180322 \\
Min. $=$ & $<0.01$ & $<0.01$ \\
Max. $=$ & 59.2 & 75.3 \\
\hline
\end{tabular}

In the table, the minimum values are less than $0.01 \mathrm{~ms}$ because of the resolution of the analysis tool at lower time values. The average interpacket delay is $1 \mathrm{~ms}$ /packet.

\subsection{Haptic Traffic Custom Probability Density Function (PDF)}

The next step is to customize the haptic network characteristics in OPNET as there is no generalised distribution that is able to represent haptic traffic distribution. The haptic distribution does not follow a fixed pattern which makes it difficult to generate analytical models of the traffic profile. Therefore, a custom PDF model was developed in order to model this haptic traffic. The inter-packet delay interval is a critical issue which is varying over time. In order to customize the haptic network model, a probability function was created.

Fig.4(a)-4(b) shows the experiment PDF that are created from the asynchronous haptic traffic data. The PDFs of both figures are obtained from over 60000 packet samples. Referring to Fig.5(a), the highest probability is 0.38 at $1.9 \mathrm{~ms}$ inter-packet time. The second highest probability is at 0.27 at an inter-packet time of less than $0.1 \mathrm{~ms}$.

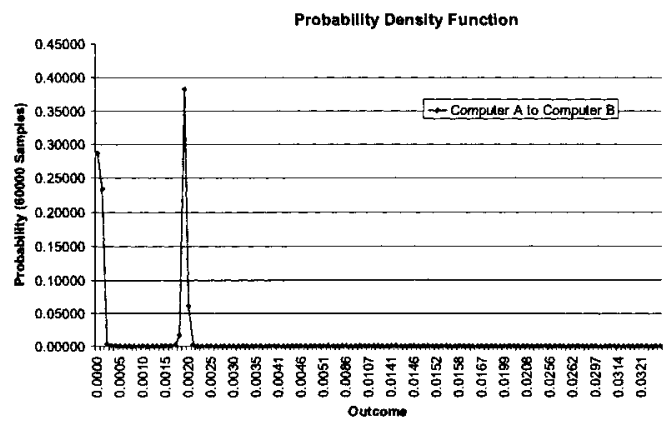

(a) 


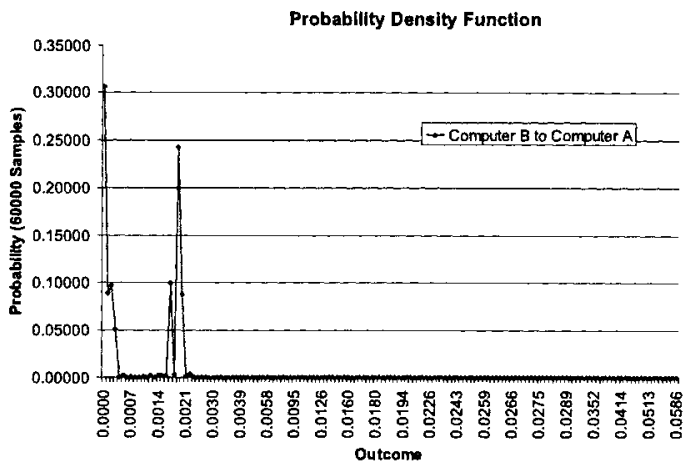

(b)

Fig. 4. Asynchronous Mode Haptic Traffic Inter-packet Time PDF Model, (a) Client to Server (Computer A to B), (b) Server to Client (Computer B to A)

In Fig.4(b), the highest probability is 0.32 at inter-packet time of less than $0.1 \mathrm{~ms}$. It is difficult to know the precise inter-packet time at this probability because of the resolution provided by the capturing tools. The second highest probability is 0.24 at $1.9 \mathrm{~ms}$. The peak (major) delays occurring in Fig. 4 are due to most of the inter-packet delays which are concentrated at $0.1 \mathrm{~ms}$ and $1.9 \mathrm{~ms}$ as shown in Fig.3.

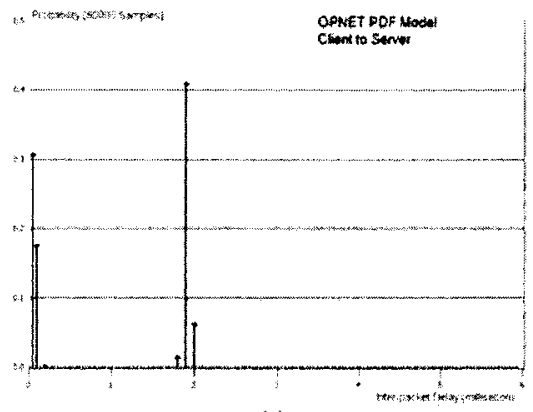

(a)

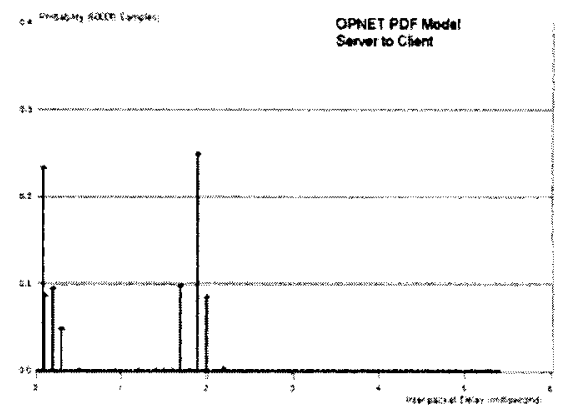

(b)

Fig. 5. Asynchronous Mode Haptic Traffic OPNET PDF Model, (a) Client to Server (Computer A to B), (b) Server to Client (Compute B to A)

The asynchronous mode probability diagrams in Fig. 4 need to be customized into OPNET PDF models as shown in Fig.5; these are created based on the experiment PDF from real world traffic by using OPNET External Model Access (EMA) code method. EMA is an OPNET term that 
defines the technique for accessing a model external to the OPNET program without using the services provided by the OPNET graphical editors. The synchronous mode PDF models which are not shown here are similar to those in Fig. 5 by using the same method discussed here. The probability and inter-packet delay values in Fig. 4 are input into an EMA code and consequently compiled to generate OPNET PDF models. The flexibility in customising the PDF model is achieved by changing content of the EMA code. In Fig.5(a), the highest probability is 0.41 at around $1.9 \mathrm{~ms}$. In Fig.5(b), the highest probability is 0.25 at $1.9 \mathrm{~ms}$ and second highest is 0.23 at time of less than 0.0001 . The higher probability in Fig.5(a)-5(b) as compare to Fig.4(a)-4(b) is due to normalization of all the probabilities in PDF model. The asynchronous mode PDF model is similar to synchronous mode PDF model but their packet sending rate is different. The asynchronous mode PDF model needs to generate a packet sending rate of 1000 packets/sec but synchronous mode PDF model needs only generates 980 packest/sec. The following sections will prove that the custom PDF models for both asynchronous and synchronous mode are able to generate haptic traffic that is closely matched to the experimental haptic traffic.

\subsection{OPNET Haptic Traffic Point-to-point Throughput}

The asynchronous mode haptic network packet size is 106 bytes without the 12 bytes of Ethernet preamble and the CRC header. Since the haptic packet transmission rate is 1000 packets/sec, thus the throughput will be $848 \mathrm{Kbit} / \mathrm{sec}$. This is closely matched to the simulation throughput of Fig.6.

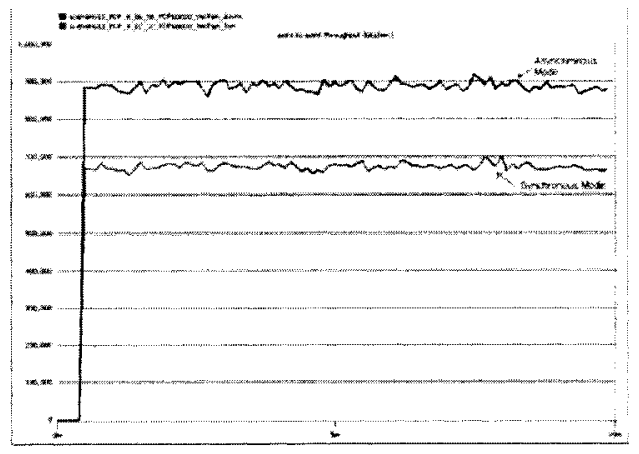

Fig. 6. Asynchronous/Synchronous Mode Custom PDF model Haptic Traffic Point-to-Point Throughput, Client to Server 
In synchronous mode, the total network packet size is 82 bytes without preamble and CRC header. Since synchronous model traffic sending rate is 980 packets/sec, the network throughput will be $627 \mathrm{Kbit} / \mathrm{sec}$; closely matched to the simulation throughput result of Fig.6.

\subsection{Distributed Haptic Virtual Network Model}

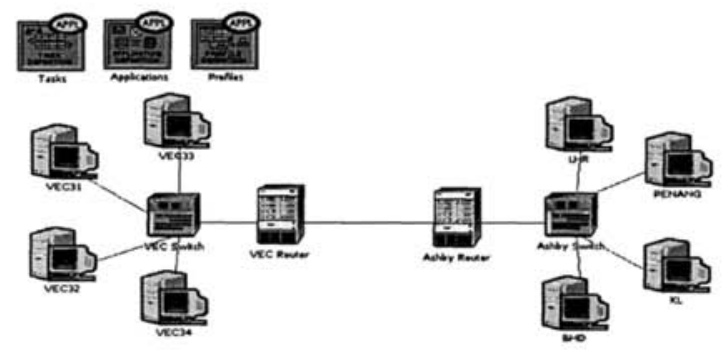

Fig. 7. OPNET Distributed Haptic Virtual Network Model

This section describes the modelling of the two haptic traffic modes in the DHVE network model. Fig.7 shows eight PCs connected peer-to-peer with two switches and routers. The two routers are connected with PPP T3 link while other links are $100 \mathrm{Mbps}$. Four haptic traffic flows are implemented between PCs VEC31-PENANG and PCs VEC32-KL respectively. The PCs VEC31/VEC33 and PENANG/LHR are configured to run a custom application task that simulates an asynchronous mode DHVE application by using the asynchronous mode PDF model created in the previous section. Additionally, the PCs VEC32/VEC34 and KL/BHD are configured to run a synchronous mode custom application by using the synchronous mode PDF model. Effectively, the DHVE network model in Fig.7 is the combination of asynchronous haptic traffic flow and synchronous haptic traffic flow. This network model has been simulated for 10 minutes by using the custom application and profile and the results are presented in Fig.8, Table 2 and Table 3.

\subsubsection{Distributed Haptic Network End-to-End Delay}

This section investigates the network characteristic under high loading of the distributed haptic network. The simulation results obtained are similar for asynchronous and synchronous modes. It can be observed that back- 
ground load above $98 \%$ results in nearly 10 seconds of end-to-end delay which is totally unacceptable for the asynchronous mode haptic operation.

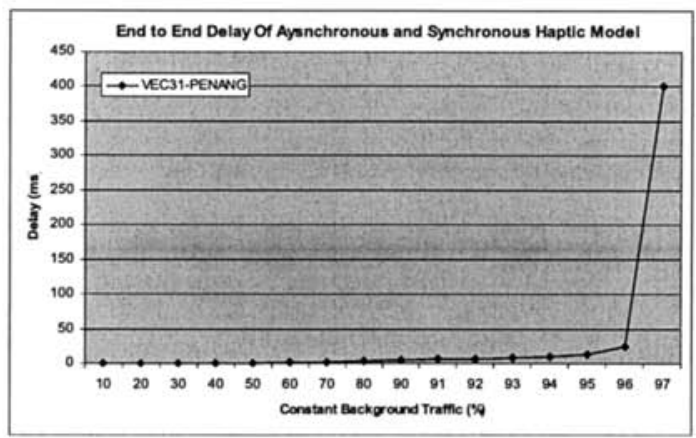

Fig. 8. Distributed Haptic Network Asynchronous/Synchronous Mode End to End Delay VEC31-PENANG(Client-Server), 10\%-97\% background load.

In Fig. 8 , the end to end delay drops to 0.02 seconds when the background decreased to $95 \%-96 \%$ but this delay is still unacceptable for haptic applications. The delay drops to $10 \mathrm{~ms}$ at $94 \%$ background loading. From the user perception experiment, a good sense of touch requires a delay of less than $5 \mathrm{~ms}$, and this occurs at less than $90 \%$ loading. These results are comparable with the results in [2], [10] which describe actual network results.

\subsubsection{Distributed Haptic Network Links Queuing Delay}

Table 2. Distributed Haptic Network Links Average Queuing Delay (ms)

\begin{tabular}{|c|c|c|c|c|}
\hline $\begin{array}{l}\text { Background } \\
\text { Loading } \\
\text { (\%) }\end{array}$ & $\begin{array}{l}\text { VEC31- } \\
\text { VEC } \\
\text { Switch (ms) }\end{array}$ & $\begin{array}{l}\text { VEC32- } \\
\text { VEC } \\
\text { Switch(ms) }\end{array}$ & $\begin{array}{l}\text { VEC Switch- } \\
\text { VEC Router } \\
(\mathrm{ms})\end{array}$ & $\begin{array}{l}\text { VEC Router- } \\
\text { Ashby Router } \\
\text { (ms) }\end{array}$ \\
\hline 0 & 0.029854 & 0.028118 & 0.0267862 & 0.015588 \\
\hline 20 & 0.88529 & 0.028154 & 0.0267589 & 0.054092 \\
\hline 50 & 0.029854 & 0.099744 & 0.1004575 & 0.211428 \\
\hline 90 & 0.220006 & 0.869559 & 1.0226898 & 2.227665 \\
\hline 95 & 2.0812 & 2.041991 & 23.976001 & 3.556528 \\
\hline 96 & 2.908303 & 2.732627 & 61.794149 & 7.137595 \\
\hline 97 & 9.658127 & 9.030557 & 214.39641 & 5.930933 \\
\hline 98 & 1520.362 & 1539.083 & 1726.272 & 6.980548 \\
\hline 99 & 1559.682 & 1560.1303 & 1669.125 & 21.18348 \\
\hline
\end{tabular}


The queuing delay represents the instantaneous measurement of packets' waiting time in the transmitter channels queue. The transmitter channels are between VEC31-VEC Switch, VE31-VEC Switch, VEC Switch-VEC Router and VEC Router-Ashby Router as shown in Fig.7. Referring to Fig.7, the asynchronous mode haptic traffic flows start from VEC31 to PENANG; the synchronous mode haptic traffic flows are from VEC32 to $\mathrm{KL}$. Therefore, the queuing delays on each particular link of the two haptic traffic flows are shown in Table 2. The results show that the queuing delays between VEC32 to VEC switch are lower than VEC31 to VEC switch simply because the synchronous mode application has a lower packet sending rate than the asynchronous application. The results also show that the major queuing occurs at the VEC Router and at the link from the VEC Switch to VEC Router. The queuing delays at the link VEC Router to Ashby Router do not increase significantly compared to the other three links.

\subsubsection{Distributed Haptic Network Effective Throughput}

Table 3. Distributed Haptic Network Effective Throughput VEC31-PENANG (Client-Server), 60\%-99\% background load (a) Asynchronous Mode, (b) Synchronous Mode.

\begin{tabular}{lcc}
\hline $\begin{array}{l}\text { Background Loading } \\
(\%)\end{array}$ & $\begin{array}{l}\text { Asynchronous Mode } \\
\text { (packets/second) }\end{array}$ & $\begin{array}{l}\text { Synchronous Mode } \\
\text { (packets/second) }\end{array}$ \\
\hline 60 & 981.957045 & 976.829825 \\
70 & 991.862847 & 973.654386 \\
80 & 999.131579 & 970.668421 \\
90 & 987.389474 & 959.100000 \\
95 & 793.389474 & 776.468421 \\
96 & 509.647368 & 502.968421 \\
97 & 77.894737 & 370.526316 \\
98 & 37.368421 & 61.578947 \\
99 & 60.000000 & 10.526316 \\
\hline
\end{tabular}

As shown in Table 3 , the haptic traffic effective throughput is reduced sharply at $97 \%-99 \%$ background traffic load because of traffic congestion starving the bandwidth available for these flows. The effective throughput is also reduced significantly at $95 \%-96 \%$. The effective throughput rate is assumed to be normal at up to $90 \%$ background traffic load. In summary, the effective throughput from each DHVE machine drops significantly above $90 \%$ background load. From the physical experiment, it was ob- 
served that at these levels, the user will feel the vibration in the PHANToM and also large abrupt force feedback. At this point the haptic system becomes unstable and the PHANTOM is not able to move further.

\section{Conclusions and Future Work}

This paper has presented a new approach to modelling haptic traffic over IP networks. The aim is to employ conventional network simulation software to simulate large-scale deployment of haptic devices and Distributed Haptic Virtual Environments (DHVEs) over the Internet. The work describes how to successfully generate a customizable haptic traffic PDF model for both synchronous and asynchronous modes of haptic interaction. This is achieved by using empirical traffic profiles obtained from a test network with haptic devices and interactive applications. Results from the simulation model show that the simulation network throughput is closely matched to experimental throughputs of $850 \mathrm{Kbit} / \mathrm{s}$ and $630 \mathrm{Kbit} / \mathrm{s}$ in asynchronous and synchronous mode respectively. The simulation results also show that DHVE effective throughput deteriorates sharply above $90 \%$ background load. In addition, end-to-end delays of more than $5 \mathrm{~ms}$ occur at above $90 \%$ background loading. Future work will include characterizing the haptic traffic under different QoS IP networks in multi-sensory environments. Differentiated Services will be incorporated into the simulation and experiment networks.

\section{Acknowledgement}

This work was supported in full by Industrial Research and Technology Unit Northern Ireland SPUR scheme, research funding of the Virtual Engineering Centre at Queen's University Belfast.

\section{References}

[1] Rima Tfaily Souayed, Gaiti, D., Pujolle, G., Wai Yu, Qiang Gu, \& Marshall, A. (2003). Haptic virtual environment performance over IP networks: a case study. Proceedings Seventh IEEE International Symposium on Distributed Simulation and Real-Time Applications, 23-25 Oct. 2003, 181-9. 
[2] Souayed R., Gaiti D. , Yu W., Dodds G., and Marshall A. "Experimental Study of Haptic Interaction in Distributed Virtual Environments", (2004) Eurohapics 2004.

[3] ZTI Telecom, http://www.zti-telecom.com/pages/main-ip.htm

[4] SenSAble Technologies. http://www.sensable.com

[5] Kessler, G. D., \& Hodges, L. F. (1996). Network communication protocol for distributed virtual environment systems. Proceedings of the IEEE 1996 virtual reality annual international symposium, mar 30-apr 3 1996, 214-221.

[6] Kyoung Shin Park, \& Kenyon, R. V. (1999). Effects of network characteristics on human performance in a collaborative virtual environment. Proceedings of virtual reality, 13-17 march 1999, 104-11.

[7] Manuel, O., Jesper, M., Joel, J., Anthony, S., \& Mel, S. Considerations in the design of virtual environment systems: A case study.

[8] Mejdi, E., Nicolas, D. G., José, R. G., \& Dimitrios, M. A scalable network architecture for distributed virtual environments with dynamic QoS over IPv6. Eighth IEEE international symposium on computers and communications.

[9] Shirmohammadi, S., \& Georganas, N. D. (2000). Collaborating in 3D virtual environments: a synchronous architecture. Proceedings of WET ICE 2000. 9th IEEE international workshops on enabling technologies: infrastructure for collaborative enterprises, 14-16 June 2000, 35-42.

[10] Tom, D., Dan, S., Maxine, B., Dave, P., Josephine, A., \& Mike, B. et al. (1999). Technologies for virtual reality/ tele-immersion applications: issues of research in image display and global networking.. EC/NSF workshop on research frontiers in virtul environments and human-centered computing, chateau de bonas, france, France.

[11] OPNET 11.0A, Product Documentation, Methodologies and Case Studies, www.opnet.com.

[12] H.G. Hoffman, T. Richard, B. Coda, A. Richards, SR. Sharar, "The Illusion of Presence in Immersive Virtual Reality during an FMRI Brain Scan", $C y$ berpsychol Behavior, vol 6, N², pp. 127-31, Apr. 2003.

[13] P. Buttolo, O. Roberto and H. Blake, "Architectures for Shared haptic Virtual Environments", special issue of Computer \& Graphics, vol. 21, No.4, PP. 421429, July-Aug 1997.

[14] S. Blake, D. Black, M. Carlson, E. Davies, Z. Wang, W. Weiss, RFC 2475, "An Architecture for Differentiated Services". 